\title{
RANDOM-REGULAR ACCELERATING STRUCTURES
}

\author{
M. Ayzatsky ${ }^{*}$, K. Kramarenko ${ }^{\#}$, NSC KIPT, Kharkov, 61108, Ukraine
}

\section{Abstract}

In this work we present results of investigation of the characteristics of inhomogeneous sections on the base of a disk-loaded waveguide with different laws of hole radius variation. We have shown that in the case when coupling of cavity fields is small there is a traveling wave in a waveguide even for the non-smooth laws of hole radius variation. But the traveling wave regime exists only at a certain frequency (operating frequency). Unlike the homogeneous accelerating structures, where reflections can arise only from the couplers, in the case of inhomogeneous structures there is no a pure traveling wave regime inside the wavegiuide at the frequencies different from the operating one. We investigate the RF properties of different accelerating structures with random distribution of the hole sizes.

\section{INTRODUCTION}

The calculations performed by us on the base of a new disk-loaded waveguide model (coupled cavity chain) [1] indicate that for waveguides with the period $D \geq \lambda / 3$, where $\lambda$ is the free-space wavelength, the "remote" coupling influences weakly on the phase shift per cell. For $\varphi=2 \pi / 3$, taking into account the "cross-cavity coupling" $((n, n-1),(n, n+1),(n-1, n+1), n$ is the cavity number) at $a / \lambda<0.14$ ( $a$ is the coupling hole radius), one can expect to achieve an accuracy of forming a phase shift per cell of the order of $\Delta \varphi=0.05$. If one restricts oneself only "paired coupling" $((n, n-1),(n, n+1))$, then, the accuracy of phase shift per cell is getting worse $\Delta \varphi=0.5$. Development of the techniques of disk-loaded waveguide cell tuning that should allow making feasible the cross-cavity coupling is a difficult task, since during tuning of the $n$-th cavity one has to take into account, somehow, the effect from the $(n+1)$-th cavity which has not yet been tuned.

In the paper [2] we present the results of our research on the technique of cell-tuning in a strongly inhomogeneous disk-loaded waveguides which realizes paired coupling.

This paper presents the simulation results of our research on the technique of cell-tuning which realizes paired coupling.

\section{UNDERLYING THEORY}

It follows from the paper [2] that an infinite chain of cylindrical cavities of the length $d$ and the radii $b_{\mathrm{n}}$, coupled through co-axial cylindrical holes with the radii $a_{\mathrm{n}}$ in the cavity dividing walls of thickness $t$ (inhomogeneous disk-loaded waveguide with the period

\footnotetext{
*aizatsky@kipt.kharkov.ua

"kramer@kipt.kharkov.ua
}

$D=d+t$ ) at $D \geq \lambda / 3$ can be, with a definite accuracy, described by a set of coupled equations

$$
\begin{aligned}
& {\left[\omega_{n}^{2}\left(1+\alpha_{n}^{(+)}+\alpha_{n}^{(-)}\right)-\omega^{2}\right] u_{n}=} \\
& \omega_{n}^{2} \beta_{n, n-1} u_{n-1}+\omega_{n}^{2} \beta_{n, n+1} u_{n+1}
\end{aligned}
$$

where $u_{n}$ is the amplitude of $E_{010}$-mode in the $n$-th cavity, $\omega_{n}$ is the $n$-th cavity eigenfrequency, $\alpha_{n}^{(-)}, \alpha_{n}^{(+)}$are the relative eigenfrequency shifts due to the coupling with $(n+1)$-th and $(n-1)$-th cavities, $\beta_{n, n-1}, \quad \beta_{n, n+1}$ are the coupling coefficients. If $\alpha_{n}^{(+)}$and $\beta_{n, n+l}$ are determined by geometrical dimensions of only the $n$-th and $(n+1)$-th cavities, as well as by the coupling hole radius $a_{n+1}\left(\alpha_{n}^{(-)}\right.$, $\beta_{n, n-1}$ are determined by geometrical dimensions of the $n$ th, $(n-1)$-th cavities and the hole radius $\left.a_{n}\right)$, then we shall say that the cavity coupling is paired. If these coefficients depend on geometrical dimensions of three cavities ( $n$-th, $(n-1)$-th and $(n+1)$-th), as well as two coupling hole radii $a_{n}, a_{n+1}$, then, such coupling we shall call "cross-cavity coupling".

Let's find the conditions when the set (1) at $\omega=\omega_{*}\left(\omega_{*}\right.$ is the operating frequency) has the solution of such form

$$
u_{n}=u_{n, 0} \exp (\operatorname{in} \varphi)
$$

where $u_{n, 0}$ is the real value. It follows from (1) that in order to achieve this the following condition is to be fulfilled

$$
\beta_{n, n-1} u_{n-1,0}=\beta_{n, n+1} u_{n+1,0}
$$

For the $n$-th cavity eq. (1) will take the form

$$
\begin{aligned}
& {\left[\omega_{n}^{2}\left(1+\alpha_{n}^{(+)}+\alpha_{n}^{(-)}\right)-\omega_{*}^{2}\right] u_{n, 0}=} \\
& 2 \omega_{n}^{2} \beta_{n, n-1} u_{n-1,0} \cos \varphi
\end{aligned}
$$

and for the (n-1)-th cavity

$$
\begin{aligned}
& {\left[\omega_{n-1}^{2}\left(1+\alpha_{n-1}^{(+)}+\alpha_{n-1}^{(-)}\right)-\omega_{*}^{2}\right] u_{n-1,0}=} \\
& 2 \omega_{n-1}^{2} \beta_{n-1, n} u_{n, 0} \cos \varphi
\end{aligned}
$$

From (2) and (3) it follows that if $\alpha_{n}^{(+)}$is independent from the parameters of the $(n+1)$-th cavity, $\alpha_{n-1}^{(-)}-$from the parameters of the $(n-2)$-th cavity and $\beta_{n-1, n}, \beta_{n, n-1}$ depend only upon the parameters of the $n$-th and $(n-1)$-th cavities, then, two equations (2) and (3) become closed and determine fully the relation of geometrical dimensions of the $n$-th and (n-1)-th cavities. In this case, having tuned the $(n-1)$-th cavity, one can find the condition, which must satisfy the geometrical dimensions of the $n$-th cavity, and, consequently, allow tuning consecutively all waveguides cavities. It can be shown 
that at the paired coupling $\beta_{n-1, n}=\beta_{n, n-1}$ and these coefficients are determined by the geometrical dimensions of the $n$-th and ( $n-1)$-th cavities, only. Things are more complicated with the dependence of coefficients $\alpha_{n}^{(+)}$on the parameters of the $(n+1)$-th cavity and $\alpha_{n-1}{ }^{(-)}$ on the parameters of the (n-2)-th cavity. Such dependence exists even under the assumption of paired coupling. However, our calculations shown that this dependence is considerably weaker than the dependence on the parameters of the $n$-th $((n-1)$-th $)$ cavity, and can be neglected, as a result.

\section{Quasi-static approach}

Let's consider the simplest model of coupling cavities that is based on the quasi-static approach [3]. We shall consider the thickness of the disk to be zero and the radius of the hole to be small. Then the coupling coefficients will have the form

$$
\begin{aligned}
& \alpha_{n}^{(+)}=\xi \frac{a_{n+1}^{3}}{b_{n}^{2} d}, \alpha_{n}^{(-)}=\xi \frac{a_{n}^{3}}{b_{n}^{2} d} \\
& \beta_{n, n-1}=\beta_{n-1, n}=\xi \frac{a_{n}^{3}}{b_{n} b_{n-1} d}
\end{aligned}
$$

where $\xi=2 / 3 \pi J_{l}^{2}\left(\rho_{01}\right), \rho_{01}$ is the first root of the zero order Bessel function $J_{0}$.

To achieve the travelling wave mode in an inhomogeneous disk-loaded waveguide with the mode type $\varphi=2 \pi / 3$ the parameters of (n-1)-th and $n$-th cavities should be connected via the relationship (it follows from Eqs. (2), (3))

$$
\begin{aligned}
& {\left[\omega_{n}^{2}\left(1+\alpha_{n}^{(+)}+\alpha_{n}^{(-)}\right)-\omega_{*}^{2}\right] \times} \\
& {\left[\omega_{n-1}^{2}\left(1+\alpha_{n-1}^{(+)}+\alpha_{n-1}^{(-)}\right)-\omega_{*}^{2}\right]=} \\
& \omega_{n}^{2} \omega_{n-1}^{2} \beta_{n, n-1}^{2}
\end{aligned}
$$

From (6) and (4)-(5) we can find

$$
b_{n}=b_{0} \sqrt{0.5\left(1+\sqrt{1+4 Z_{n}}\right)}
$$

where $b_{0}=c \rho_{01} / \omega_{*}$,

$$
\begin{aligned}
& Z_{n}=\xi \frac{\left(a_{n}^{3}+a_{n+1}^{3}\right)}{b_{0}^{2} d}-4 \cos ^{2} \varphi \xi^{2} \frac{a_{n}^{6}}{b_{0}^{2} b_{n-1}^{2} d^{2}} \times \\
& {\left[1-b_{n-1}^{2} / b_{0}^{2}+\xi\left(a_{n-1}^{3}+a_{n}^{3}\right) /\left(b_{n-1}^{2} d\right)\right]^{-1}}
\end{aligned}
$$

Suppose we have placed the $n$-th and (n-1)-th cavity into some sort of a cavity stack. It can be shown that the condition (6) is fulfilled in the case, when in the cavities, adjoining the cells under consideration, the amplitudes of $E_{010}$ - modes equal to zero. Such cavity stacks have already been used for tuning separate parts of quasiconstant impedance sections for LIL accelerator [4]. However, there the cells were tuned not consecutively, i.e. beginning from the entrance (or exit), but in different stacks being then simply joined one-to-one.

\section{Stability of tuning technique}

Our results [2] indicate that it is possible to use a consecutive tuning of all cells for disk-loaded waveguide with an arbitrary law of the coupling hole radius variation. With that, at the operating frequency $\omega=\omega_{*}$ the travelling wave mode with the phase shift of the order of $2 \pi / 3$ with a certain accuracy is guaranteed in a waveguide.

Consequtive tuning feasibility is determined by the stability of this technique. Our numerical analysis show that small errors in the tuning of individual cells should not lead to the exponential growth of subsequent deviations, i.e. the technique is stable.

\section{RANDOM STRUCTURE}

Using such tuning technique we can consider accelerating structures with various laws of the hole radius variation, which will operate in a travelling wave mode at $\omega=\omega_{*}$.

We give the random deviations of the hole sizes in the constant impedance accelerating structure $(a=1.25 \mathrm{~cm})$. The random sizes of the holes are uniformly distributed in the interval $1.25 \pm 0.05 \mathrm{~cm}$. The cavity radii and the parameters of the couplers are tuned depending on the hole sizes and operating frequency $f_{*}=2.7972 \mathrm{GHz}$. The cavity radii are tuned according to the considered technique. The cavity length is $d=\lambda / 3$. We tune the structure by such a way that the phase shift per cell equals $2 \pi / 3$. So, we can consider such structure as random regular accelerating structure.

Let's compare the random-regular accelerating structure with the constant impedance one, which already has been tuned according to the described above technique (see Fig. 1).


Figure 1: constant impedance structure - a) phase shift per cell; b) reflection coefficient $R=P^{(-)} / P_{0}$ versus the frequency. 
In the random-regular structure the phase deviation from the phase shift per cell $\varphi_{0}=2 \pi / 3$ is small $-\approx 0.5^{\circ}$ and the amplitudes of electric field take strongly different values in various cells (see Fig. 2). The energy gain in such random - regular structure is even a bit greater then in constant impedance one.

In the case of constant impedance structure reflection can arise only from the couplers within the passband (see Fig. 1b). Unlike the constant impedance structure, in the random-regular structure there is no pure traveling wave regime at the frequencies different from the operating one (see Fig. 3).
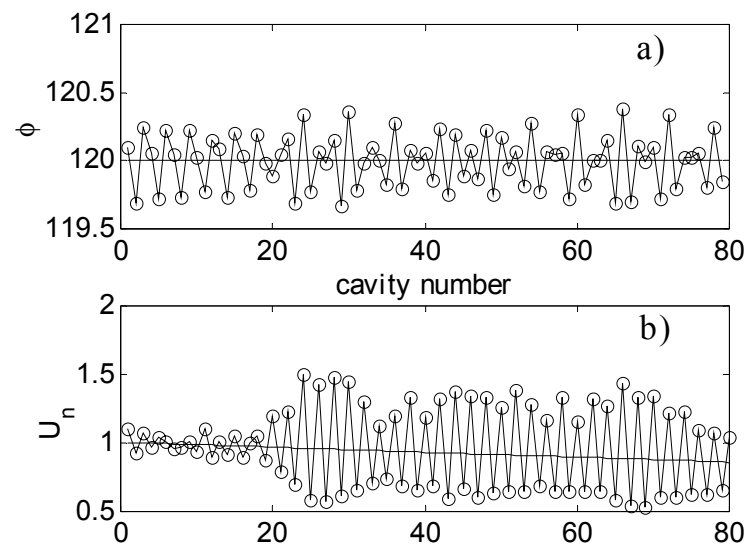

Figure 2: random (constant impedance) structure a) phase shift per cell; b) amplitude distribution.

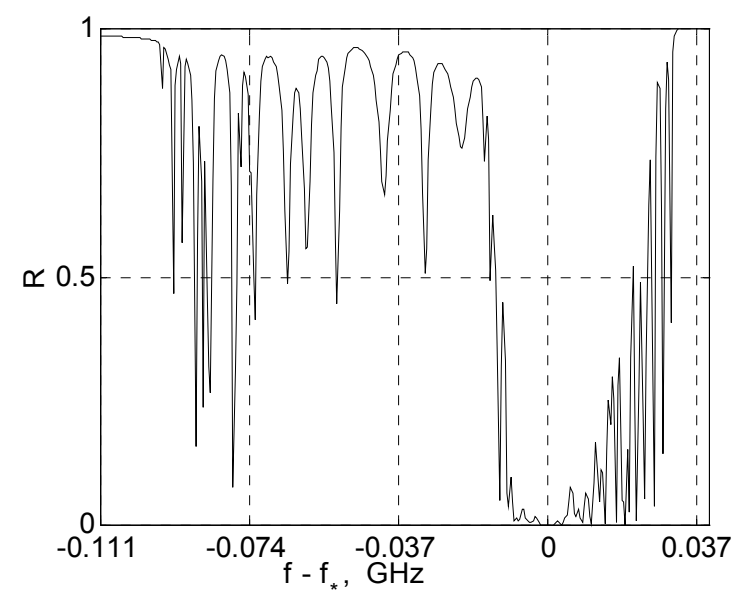

Figure 3: reflection coefficient $R=P^{(-)} / P_{0}$ in the random (constant impedance) structure versus the frequency.

The constant gradient accelerating structure is more useful in comparison with the constant impedance one. The radii of the holes in the constant gradient structure decrease from entrance to exit. They decrease by the value $0.002 \mathrm{~cm}$ from cell to cell. We give the random deviations of the hole sizes in such constant gradient accelerating structure. As in the previous case, the random sizes of the holes are uniformly distributed in the interval $\pm 0.05 \mathrm{~cm}$. The characteristics of such random- regular accelerating structure are shown in figures 4 and 5 .
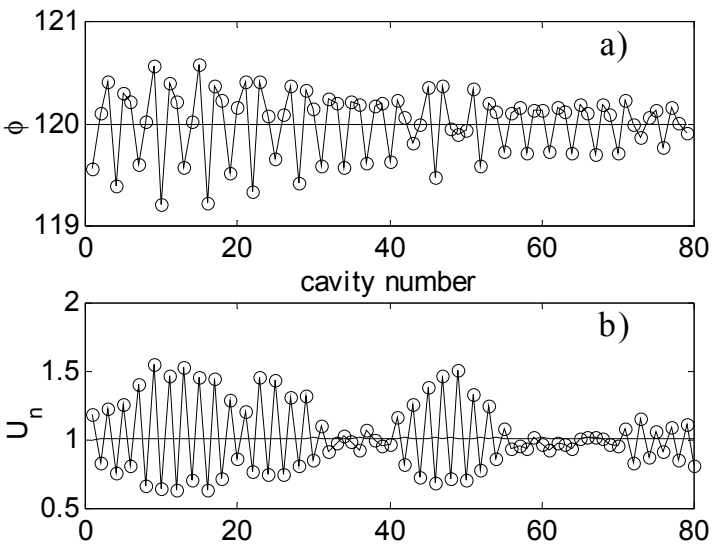

Figure 4: random (constant gradient) structure - a) phase shift per cell; b) amplitude distribution.

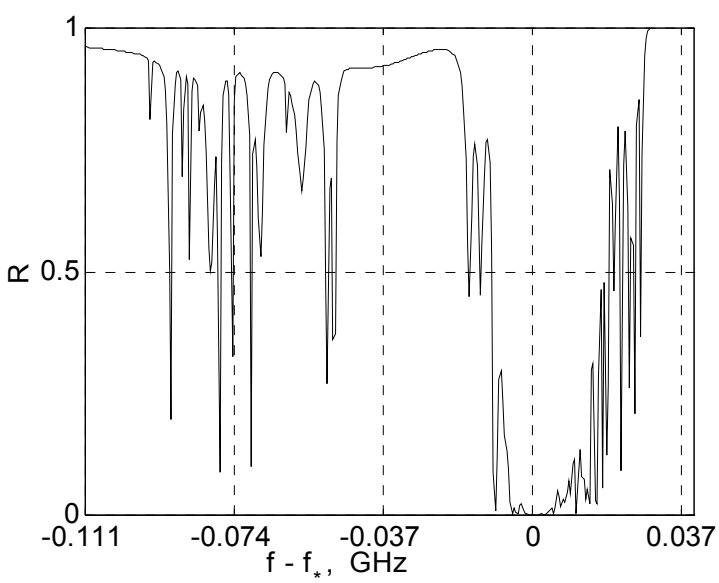

Figure 5: reflection coefficient $R=P^{(-)} / P_{0}$ in the random (constant gradient) structure versus the frequency.

There is "detuning" of the first dipole passband in the considered random-regular structures. These structures can find application in high current accelerators and in the travelling wave klystron output circuits.

\section{REFERENCES}

[1] M.I. Ayzatsky, "New Mathematical Model of an Infinite Cavity Chain", EPAC'96, Sitges, June 1996, p. 2026.

[2] M.I. Ayzatsky, E.Z. Biller, "Development of Inhomogeneous Disk-Loaded Accelerating Waveguides and RF-coupling", LINAC'96, Geneva, August 1996, p. 119.

[3] H.A. Bathe, Phys. Rev. 66 (1944) 163.

[4] G. Bienvenu, J.C. Bourdon, P. Brunet et. al., "Accelerator Structure Developments for the LEP Injector Linac”, LINAC'84, Seeheim, 1984. 